\title{
Papel de los profesionales de la salud en el diseño, obtención y entendimiento del consentimiento informado: Una revisión
}

\section{Role of health professionals in the design, obtention and understanding of informed consent: $A$ review}

\author{
Andrés Camargo ${ }^{1}$; Jessica Olmos ${ }^{2}$; Elkin Higuera-Dagovett'; Rafael Vargas ${ }^{4}$; Ruth Barreto ${ }^{5}$
}

\begin{abstract}
${ }^{1}$ Enfermero,M.Sc.UniversidaddeCienciasAplicadasyAmbientalesU.D.C.A,FacultaddeCienciasdelaSalud,ProgramadeMedicina;e-mail:andcamargo@udca.edu.co; (Dhttps://orcid.org/0000-0003-4723-9310
\end{abstract}

²Enfermera. Universidad de Ciencias Aplicadas y Ambientales U.D.C.A, Facultad de Ciencias de la Salud, Programa de Enfermería; e- mail: jeolmos@udca. edu.co; (Dhttps://orcid.org/0000-0003-4073-0051

${ }^{3}$ Médico Cirujano, Especialista, M.Sc. Universidad de Ciencias Aplicadas y Ambientales U.D.C.A, Facultad de Ciencias de la Salud, Programa de Medicina; e-mail: elhiguera@udca.edu.co; Dhttps://orcid.org/0000-0002-2730-5731

${ }^{4}$ Médico Cirujano, M.Sc., Ph.D. Universidad de Ciencias Aplicadas y Ambientales U.D.C.A, Facultad de Ciencias de la Salud, Programa de Medicina. Universidad Antonio Nariño, Facultad de Medicina; e-mail: rvargas3200@hotmail.com; Dhttps://orcid.org/0000-0001-5702-9240

${ }^{5}$ Enfermera,M.Sc.UniversidaddeCienciasAplicadasyAmbientalesU.D.C.A,FacultaddeCienciasdelaSalud,ProgramadeEnfermería;e-mail:rbarreto@udca.edu.co; (Dhttps://orcid.org/0000-0001-9867-641X

Cómo citar: Camargo, A.; Olmos, J.; Higuera-Dagovett, E.; Vargas, R.; Ruth Barreto, R. 2019. Papel de los profesionales de la salud en el diseño, obtención y entendimiento del consentimiento informado: Una revisión. Rev. U.D.C.A Act. \& Div. Cient. 22(2):e1164. http:// doi.org/10.31910/rudca.v22.n2.2019.1164

Artículo de acceso abierto publicado por Revista U.D.C.A Actualidad \& Divulgación Científica, bajo una licencia Creative Commons CC BY-NC 4.0

Recibido: Febrero 8 de 2019

Aceptado: Septiembre 18 de 2019

Editado por: Ingeborg Zenner de Polanía

\section{RESUMEN}

El consentimiento informado es un proceso que tiene la intención de autorizar o llegar a un acuerdo con el profesional para la realización de un procedimiento en el área de la salud o en el campo de la investigación, que se formaliza con la firma de un documento. Este consentimiento refleja la voluntad y la autonomía de dicho individuo y su capacidad, una vez ilustrado en detalle sobre la intervención, para entender los resultados principales y los potenciales riesgos de dicha intervención y su voluntad de asumirlos, sin presión externa. Aunque esto parece trivial, la historia muestra que voluntad y autonomía no han sido valores tenidos en cuenta en el área de la salud y menos en el campo de la investigación, de manera intencional o por omisión. Para la presente revisión, se realizó una búsqueda bibliográfica en las bases de datos PubMed, Medline, Scielo y Google Académico. Se utilizaron los siguientes descriptores: historia de la investigación, ética, consentimiento informado, investigación, enfermería y comprensión, encontrando 2.800 artículos, en idiomas inglés, portugués y español, sin restricción de un límite de tiempo; luego, se efectuó la lectura del resumen de los artículos, seleccionando los 80 que más se acercaban al tema y se emplearon. Como resultado del análisis de la información, se generaron algunas pautas orientadas a mejorar el proceso de consentimiento informado, a través de técnicas que mejoren la explicación por parte del profesional de salud y la comprensión del participante o paciente. 
Palabras clave: ética; consentimiento informado; historia; práctica; investigación

\section{ABSTRACT}

Informed consent is a legal document signed by an individual who will undergo an intervention in the health area, or in the research field. This informed consent reflects the individual wellbeing and autonomy and his / her capacity, once he or she has been illustrated in detail about the intervention, to understand its main results and its potential risks of said intervention and his / her willingness to assume it without external pressure. Although this seems trivial, history shows that wellbeing and autonomy have not been considered values in the health area and lesser in research, intentionally or by omission. For the present review, a bibliographic search was carried out in PubMed, Medline, Scielo and Google Scholar databases. The following descriptors were used: history of research, ethics, informed consent, research, nursing and comprehension. We found 2800 articles, in English, Portuguese and Spanish, without time limit. Then after reading abstract of the Articles it was selected 80 articles that were close to the subject and there were used. As a result of the analysis of the information, some guidelines were generated aimed to improve the informed consent process through techniques that improve the explanation by the health professional and the understanding of the participant or patient.

Keywords: ethics; Informed consent; history; practice; research.

\section{INTRODUCCIÓN}

Aunque el consentimiento informado (CI) podría ser entendido por los profesionales de la salud como una "carga" administrativa que finaliza con la firma de un "documento" (Kottow, 2016), existe consenso con relación a que es un proceso que tiene la intención de autorizar o llegar a un acuerdo con el profesional, para la realización de un procedimiento, donde se aclararan los riesgos y otras alternativas de tratamiento que, además, se debe basar en los principios de autonomía y autodeterminación del paciente (AlSaadoon \& Al-Adawi, 2019; Simkulet, 2017; Cañete et al. 2012). En ese sentido, el CI es un proceso comunicativo interpersonal, que involucra la capacidad del profesional para comprender y acompañar al paciente, de tal forma, que le permita tener claras las implicaciones del procedimiento o tratamiento al que va a ser sometido, para la toma de decisiones (Kottow, 2016).

\section{Aspectos históricos del consentimiento informado: Sus} precedentes remontan a la Grecia antigua, cultura para la que era importante que los hombres libres tuviesen la posibilidad de decisión para aceptar o no un tratamiento médico, lo que da cuenta, de los conceptos de autodeterminación y autonomía, inmersos en el concepto de libertad, entendida como la capacidad de decisión de los seres humanos, incluida en el pensamiento moderno (Chuaire \& Sánchez, 2007). En Estados Unidos, a principios del siglo XX, se presentaron varios casos, en los que la decisión del juez fue a favor de los pacientes, sin cuestionar el conocimiento y el acto médico en sí, sino que catalogó la conducta como una agresión, al no tener en cuenta la decisión de los pacientes tratados, lo que da cuenta, nuevamente, de los conceptos de autodeterminación y autonomía (Chuaire \& Sánchez, 2007). Uno de los casos es el de Ana Mohr, en 1905, quien perdió su capacidad auditiva posterior a habérsele realizado una intervención quirúrgica en el oído contrario al que había autorizado, porque, según el concepto del médico, era conveniente intervenirlo primero (Nelson-Marten \& Rich, 1999; Chuaire \& Sánchez, 2007).

En ese sentido, la historia ha mostrado que el derecho de los pacientes a dar su consentimiento está por encima de la intención del acto médico en sí, concepto que trasciende los contextos clínicos y adquiere fuerza en los contextos de investigación, excepto en situaciones particulares en las que está permitido omitir el CI como: atención en servicios de urgencias, cuando el paciente necesita tratamiento inmediato para salvaguardar la vida y ciertas intervenciones en salud pública (Lin et al. 2019).

A finales del siglo XIX, Albert Ludwig Sigesmund Neisser, profesor de dermatología y venereología en la universidad de Breslau, Alemania, realizó ensayos clínicos con personas, trabajadoras sexuales en su gran mayoría, a quienes inyectaba sueros acelulares de pacientes con diagnóstico de sífilis, con el objetivo de prevenir la enfermedad (Vollman \& Winau, 1996). Aunque estos ensayos no cumplieron con el objetivo y las explicaciones acerca del porqué algunos de los sujetos en experimentación habían presentado sífilis estaban sustentadas en el hecho de que eran trabajadoras sexuales, argumento que fue apoyado, en su mayoría, por la comunidad médica. Neisser fue multado por el Real Tribunal Disciplinario, argumentando que no se solicitó el consentimiento a los participantes del ensayo (Vollman \& Winau, 1996). De nuevo, como en los casos referenciados por Chuaire \& Sánchez (2007), no se cuestiona el conocimiento ni el acto médico en sí, sino la no consideración en el acto de la libertad, que tienen las personas de ser informadas y de elegir.

Los casos más cuestionados de investigación médica en el siglo XX, se presentaron en los años 40 , durante la segunda guerra mundial. Es importante anotar que antes del período de la Alemania Nazi, ya existían documentos que daban recomendaciones para las terapias e investigación en seres bumanos, sumados a los antecedentes en contextos jurídicos de casos que habían fallado a favor de los pacientes y ponían límites, no solo a las intervenciones sino a los procesos de investigación en humanos (López-Muñoz et al. 2008; Vollman \& Winau, 1996). En ese sentido, no solo se utilizaron como sujetos de experimentación ciudadanos no arios, judíos y prisioneros de guerra en campos de concentración (Bogod, 2004; Sabbatani, 2013; Silver, 2011), sino que existían recomendaciones de esterilización y de eliminación de aquellas personas con alguna condición médica, que pusiera en peligro la supremacía de la raza Aria (López-Muñoz et al. 2008; González-López \& Ríos-Cortés, 2016; Sabbatani, 2013). Así como en la Grecia antigua, los esclavos, a diferencia de los hombres libres, no tenían el derecho a ser informados acerca de los tratamientos a realizar (Chuaire \& Sánchez, 2007); en la Alemania Nazi, las teorías científicas raciales que alimentaban las ideas del Partido Nacionalista Obrero Alemán permitieron que, en el contexto 
de investigación, los beneficios del Estado estuvieran por encima de los derechos del paciente (López-Muñoz et al. 2008; GonzálezLópez \& Ríos-Cortés, 2016).

Con la finalización de la segunda guerra mundial, se hicieron públicos los vejámenes cometidos por militares y científicos alemanes, que culminó con los llamados Juicios de Nuremberg, en donde se declararon culpables, por crímenes de guerra contra la humanidad, a siete médicos alemanes, involucrados en investigaciones médicas, dando como resultado, posteriormente, al Código de Nuremberg, que da elementos éticos y legales para la experimentación en seres humanos, que incluye, entre otros, el consentimiento previo de los sujetos en experimentación (Katz, 1996; Annas, 2018; Dhai, 2014).

La investigación médica en Estados Unidos: Por solo citar algunos ejemplos, el "Estudio Tuskegee", realizado por investigadores del Servicio Público de Salud de Estados Unidos, entre 1932 y 1972, en Tuskegee (Alabama), tenía como objetivo describir los efectos de la sífilis no tratada (Fairchild \& Bayer, 1999). Para esto, se utilizaron afroamericanos de escasos recursos económicos con diagnóstico de sífilis, quienes se dejaron en observación durante décadas, sin informarles que tenían el diagnóstico y sin iniciar ningún tratamiento, a pesar de que existía; además, fueron engañados para que hicieran parte de la investigación (Fairchild \& Bayer, 1999; Sandvik \& Lie, 2016). El mismo Servicio Público de Salud de Estados Unidos realizó en Guatemala, con el apoyo del gobierno, un estudio experimental, con personas en condición de vulnerabilidad, como niños, huérfanos, trabajadoras sexuales menores de edad, indígenas, entre otros, quienes fueron infectados con neisseria gonorrea, treponema pallidum y no se les informó el objetivo del estudio (Rodríguez \& García, 2013; Reverby, 2016).

Del código de Nuremberg, al Informe Belmont y al nacimiento de la bioética: En el seno de la sociedad, se han hecho esfuerzos importantes para generar reglas que rijan la investigación con seres humanos, lo cual, se hizo evidente al final de la segunda guerra mundial, con el Código de Nuremberg, que sirvió de base para la Declaración de los Derechos Humanos, entre otros (Dhai, 2014). En este código, se plantean tres elementos básicos para investigación en humanos: i) el consentimiento informado voluntario, ii) el análisis de riesgo/beneficio y iii) el derecho a retirarse del estudio sin repercusiones (disentimiento), elementos que sirvieron como fundamento de los posteriores códigos de ética (Vollmann \& Winau, 1996). En Paris, 1948, la Asamblea General de las Naciones Unidas aprueba el documento "Declaración Universal de los Derechos Humanos", un marco ético que se crea con el fin de garantizar la justicia y la igualdad de todos los seres humanos, que sirve como garante para los individuos que participen en las investigaciones médicas (Rice, 2008). Posteriormente, en 1964, la Asociación Médica Mundial (AMM) divulga una propuesta de principios éticos para investigación médica en seres humanos, llamada "Declaración de Helsinki”, estableciendo, detalladamente, los deberes éticos al momento de realizar investigación clínica, basándose en el Código de Nuremberg y la declaración de Ginebra. La Declaración de Helsinki, se centra en: i) la protección de personas vulnerables, ii) la relación riesgo/beneficio favorable, iii) el consentimiento informado (Declaración Universal de los Derechos Humanos, 1948; Declaración de Helsinki, 2013); sin embargo, la principal preocupación, está en que la declaración de Helsinki en sus siete revisiones desde 1964 hasta el 2013, esté siendo permeada por los intereses particulares de investigadores y patrocinadores de las investigaciones, en detrimento de su objetivo inicial, de buscar el bienestar de los sujetos de investigación, por encima de intereses particulares (Kottow, 2014; Manzini, 2000).

Como colaboración con la OMS, el Consejo de Organizaciones Internacionales de las Ciencias Médicas (CIOMS) publica el documento "Propuesta de Pautas Internacionales para la Investigación Biomédica en Seres Humanos", que buscan establecer guías para la aplicación de los principios establecidos en las declaraciones anteriormente divulgadas en los países en desarrollo, teniendo en cuenta la situación cultural y socioeconómica de los mismos. Hacia 1978, como resultado del juicio realizado con relación al estudio Tuskegee, se genera el informe de Belmont, por parte de la Comisión Nacional para la Protección de los Sujetos Humanos ante la Investigación Biomédica y del Comportamiento, que define los principios éticos fundamentales aceptados como los requisitos mínimos para la investigación ética en seres humanos, como lo son: 1) La beneficencia, que pretende que la investigación se plantee para maximizar los beneficios y minimizar el daño; 2) La justicia, en cuanto a la distribución del riesgo por igual a las personas que participen de la investigación y 3) El respeto a las personas que abarca uno de los aspectos más importantes, como lo es la autonomía como derecho de cada individuo a la autodeterminación, es decir, la libertad de cada uno a elegir si participa o no en la investigación, lo cual, se aborda, en gran medida, con el CI. De esto surgen tres aplicaciones prácticas en investigación: 1) El consentimiento informado, 2) La evaluación del balance riesgo/beneficio y 3) La selección adecuada de sujetos de estudio (Sims, 2010).

Finalmente, en los años 70, surge la bioética. Aunque aparentemente este término no es nuevo (Sass, 2007), solo fue hasta 1970, cuando Van Rensselaer Potter, un bioquímico norteamericano investigador de cáncer, popularizó el término (Potter, 1970). Esto dio origen a una nueva disciplina, la Bioética, que ha tomado las riendas en términos de regulación de la práctica clínica y la investigación médica, apoyada en cuatro principios básicos: 1) Beneficencia, 2) no-maleficencia, 3) justicia y 4) autonomía (Beauchamp \& Childress, 2009).

\section{MATERIALES Y MÉTODOS}

Para el presente trabajo, inicialmente, se realizó una revisión bibliográfica en bases de datos, como PubMed, Mediline, Scielo y Google Académico, tomando de base descriptores como: historia de la investigación, ética, consentimiento informado, investigación, enfermería, medicina y comprensión. Se encontraron 2.800 artículos, en idioma inglés, portugués y español, sin restricción de un límite de tiempo; se procedió a la lectura del resumen de los artículos y se seleccionaron 80 artículos, los cuales, están relacionados al tema. Se emplearon en forma conjunta, para que los autores, expertos en el tema de investigación y ética, derivado de su amplia experiencia en el área de investigación básica y aplicada en modelos animales 
y en seres humanos, evaluación de proyectos a nivel de pregrado y posgrado; consultorías, evaluaciones y supervisión de proyectos para agencias gubernamentales, realizaran el análisis.

\section{RESULTADOS Y DISCUSIÓN}

Como resultado del análisis y discusión de los diferentes aspectos presentados en los artículos analizados, se desglosaron tres tópicos fundamentales, que pueden ser materia de discusión permanente con relación al CI y la investigación: 1) Comprensión del CI, 2) Regulación institucional del CI escrito y 3) CI, práctica clínica e investigación: el papel del profesional de la salud.

Comprensión del consentimiento informado: En cada uno de los escenarios en que es aplicado el CI, se pueden presentar barreras para su comprensión (Suárez-Obando, 2016). En el caso de personas que no hablan el mismo idioma, el lenguaje técnico del profesional de la salud podría posibilitar que la información sea distorsionada o tergiversada (Lad \& Dahl, 2014; Fernandes, 2015). En ese sentido, en diferentes estudios, se han utilizado la traducción del documento e intérpretes, lo que ha facilitado la comunicación entre las dos partes y el entendimiento de este por los participantes (Karliner et al. 2007). Esto permite, que se comprenda la información brindada en el consentimiento, se respete la autonomía del paciente y que tenga la posibilidad de decidir voluntariamente (Karliner et al. 2007); sin embargo, si el intérprete no es un profesional de la salud, sus comprensiones acerca del documento podrían incidir de forma inadecuada en la percepción del paciente frente a su intervención o el objetivo de la investigación (Hunt \& de Voogd, 2007; Hadziabdic et al. 2011).

En ese sentido, es importante la percepción de quien aplica el CI y la del paciente o de la persona que hará parte de la investigación, de tal forma, que se pueda generar una mejor comprensión. Este aspecto toma mayor fuerza cuando se trabaja con personas en contextos culturales y sociales específicos como, por ejemplo, comunidades indígenas, poblaciones marginadas o con nivel educativo bajo, ya que su percepción de lo que es estar sano o enfermo podría contener elementos difíciles de entender para el profesional de la salud, lo que dificulta la comunicación entre las partes (Halkoaho et al. 2016).

La capacidad de los participantes potenciales de consentir voluntariamente para la investigación también depende del empoderamiento y la autonomía para la toma de decisiones, que implica variables, como el momento vital y el desarrollo cognitivo. En los niños, por ejemplo, la toma de decisiones queda a cargo de los padres; sin embargo, son partícipes de su tratamiento, a través de su asentimiento (Khabour et al. 2017), que se puede obtener implementando diferentes métodos de consentimientos didácticos, como cine foros, historietas o comics, en donde los niños pueden conocer el tratamiento que les van a realizar, los riesgos, los beneficios y, finalmente tener un espacio en donde puedan expresar si quieren participar o no (Grootens-Wiegers et al. 2015).

Es importante tener en cuenta que en el grupo de adultos mayores se podrían presentar con mayor frecuencia alteraciones cognitivas relacionadas con el envejecimiento o con enfermedades prevalentes en este grupo (Prusaczyk et al. 2017). La evidencia actual sugiere que la corteza prefrontal guía la toma de decisiones en un entorno social complejo y las medidas del funcionamiento ejecutivo y de la fluidez de la palabra (trayectoria y recuerdo inmediato), así como las habilidades de atención son los predictores más importantes de la capacidad para las decisiones de CI (Porrino et al. 2015). En ese sentido, se ha observado que a mayor deterioro cognitivo menor es la comprensión de la información (Porrino et al. 2015; Escudero Carretero et al. 2017) y, en algunos casos, se suman las demencias, que aumentan la vulnerabilidad, entendida como el riesgo de explotación y la capacidad limitada para comprender la información para tomar una decisión (West et al. 2017).

Otro aspecto importante es el grado de educación de los participantes; entre mayor grado de escolaridad mejora el entendimiento del CI (Breese et al. 2007). Muchas personas que solo cuentan con primaria consideran que el consentimiento informado es la firma de un documento que tiene información brindada por un profesional en salud y otros no saben qué es un CI, limitándolo solo a documentos (Escobar López \& Carrera Celis, 2015). Las personas con grados medios de educación señalan que el CI es la forma de aceptar una información brindada por un profesional de la salud firmando un documento y, quienes han alcanzado un nivel más alto de educación, refieren que es una toma de conciencia frente a una información brindada por parte del profesional de la salud y requiere la toma de decisiones con responsabilidad sobre una intervención (Escobar López \& Carrera Celis, 2015; Guix Oliver et al. 1999).

Técnicas para mejorar la comprensión del consentimiento informado: A continuación, se enuncian las principales técnicas que reporta la literatura para mejorar la comprensión del CI, evaluando los pros y los contras y de acuerdo con las particularidades de la investigación, los investigadores, los profesionales de la salud y sus recursos económicos.

Uso de videos y herramientas multimedia. Es una estrategia que ha demostrado resultados positivos en población sana y con problemas mentales. Con el desarrollo de la tecnología han disminuido su costo y la dificultad de su diseño e implementación, evidenciando un aumento en la comprensión del CI y la retención de la información semanas después (Carreño-Dueñas, 2016), incluso, en personas con un bajo nivel de escolaridad y conocimiento limitado de intervenciones médicas (Karan et al. 2014), lo que parece que puede influir en el deseo de la persona para participar en un estudio a corto plazo y entender los procedimientos a realizar en las intervenciones clínicas. Investigaciones con pacientes que van a ser sometidas a laparatomía laparoscópica (Ellet et al. 2014), padres de niños que van a ser sometidos a sedación con ketamina (Spencer et al. 2015), entre otras, han mostrado que complementar con una estrategia multimedia el proceso del CI mejora el entendimiento acerca del procedimiento; no obstante, para algunos autores se necesita generar mayor evidencia del impacto de este tipo de estrategias en el entendimiento y la ansiedad, en la participación en ensayos clínicos (Synnot et al. 2014). 
Interacción persona-persona. Es una técnica que permite hacer una discusión ampliada de las intervenciones que se van a realizar y puede mejorar notablemente la comprensión del CI; es la técnica más utilizada en investigación que, en la práctica, permite explicar, de manera más extensa, la naturaleza del estudio y de la intervención $y$, de esta manera, estar más satisfechos con la información (Ssali et al. 2015); sin embargo, dentro de las dificultades que se tienen es el tiempo y los costos extras para el estudio, al requerir un profesional exclusivamente para esta actividad.

Redacción y construcción del consentimiento informado. El CI, se debe escribir en un lenguaje sencillo, teniendo en cuenta el grado de escolaridad y la facilidad de lectura de gramática pasiva (Flory \& Emanuel, 2004). Se debe tener en cuenta la complejidad del texto, donde se incluye, la longitud del párrafo, el número de palabras por oración y el número de caracteres de las palabras; la longitud teniendo en cuenta los caracteres, oraciones, párrafos y el número de páginas, se puede subrayar lo que se crea importante (Beskow et al. 2010).

Dependiendo de la complejidad de la investigación o la intervención clínica, el documento puede llegar a un promedio de 20 páginas, con un rango entre 14 y 27 (Malik \& Mejia, 2014). Es importante tener en cuenta que a mayor número de páginas se podría disminuir la comprensión del documento, en ese sentido, se mostró una mejora en la comprensión del CI al disminuir su extensión de 4 a 2 páginas, mediante la eliminación de la información no pertinente (Rogers et al. 1998).

Evaluación de la comprensión. Se puede evaluar la comprensión por medio de breves cuestionarios de selección múltiple o entrevistas cortas con el participante. Para unos autores es más útil y sencillo dividir el proceso de CI en cuatro pasos, iniciando con la presentación de la información de la manera más clara y sencilla para el participante, seguido de la comprensión de asuntos importantes, en donde se hace énfasis a los aspectos más relevantes de la investigación o intervención; luego, viene la decisión voluntaria y reflexiva, en donde al participante se le plantea todas sus alternativas y, por último, lo comunica a los investigadores, recalcando aquí que la mejor alternativa es dejar el documento escrito, entregando una copia para que se dirijan al documento si tienen alguna duda (Hoover-Regan et al. 2013; Malik \& Mejia, 2014; Carreño Dueñas, 2016).

Regulación legal del CI: El consentimiento, se formaliza a través de un documento escrito, donde el participante o paciente firma, dando fe de que ha entendido y que, de manera autónoma, acepta participar en el estudio o acepta la realización de un procedimiento o una intervención. Aunque el CI obedece a códigos internacionales es necesario conocer las regulaciones legales de cada país. En Colombia, según la resolución 8430 de 1993 del Ministerio de Salud, debe contener información acerca de: (i) el objetivo de la investigación o del procedimiento clínico a realizar, (ii) los riesgos a los que se está expuesto y la forma como se van a minimizar, (iii) los beneficios de la investigación o del procedimiento, (iv) la forma como se van a tratar y manejar los imprevistos, (v) la forma como se garantiza la confidencialidad de la información, (vi) la documentación que soporte la investigación o el procedimiento clínico y (vii) la voluntad para participar. Adicionalmente, es importante tener en cuenta que el riesgo en investigación con humanos no solo se debe limitar al riesgo físico o psicológico, sino al riesgo en el manejo de datos y de información que pueda afectar al participante, por eso es relevante tener en cuenta, en Colombia, regulaciones adicionales, como son la ley estatutaria 1581 de 2012 y el decreto 1377 de 2012.

Para obtener el consentimiento informado de un participante en un proceso de investigación, deben estar presentes cuatro elementos, para que sea moralmente aceptable y valido: 1) Competencia (tanto la capacidad del profesional para llevar a cabo su intervención como de la persona para participar en la misma); 2) información relacionada con la investigación o procedimiento que se va a realizar; 3) capacidad de entender y de analizar la información suministrada (comprensión) y 4) libre elección para participar en la investigación (voluntariedad), con el fin de generar un proceso, mediante el cual, se respeta el principio de autonomía del paciente (Vera, 2016).

Consentimiento informado, práctica clínica e investigación. El papel del profesional de la salud: La cultura de la atención en salud encierra los derechos de los pacientes y los mueve a ser socios activos de su salud, por lo tanto, el CI deja de ser el logro de la obtención una firma y ha pasado a ser un proceso, que involucra varios aspectos, centrados en el respeto del derecho de cada persona, al momento de decidir (Clifford, 2013; Menendez, 2013), reforzado mediante el entendimiento de los profesionales, en cuanto a lo ético y lo jurídico que esto implica y el deber y la responsabilidad que se tiene al momento de iniciar o dirigir una intervención o investigación.

El profesional de la salud está para apoyar a la persona, integrar los conocimientos que tiene acerca de lo que se va a realizar y presentarlos de una manera clara, basándose no solo en el deseo del profesional sino también en la consideración cuidadosa de los principios éticos, de tal manera que le facilite al participante la toma de decisiones, convirtiéndose el $\mathrm{CI}$ en un proceso dinámico de intercambio entre el paciente y el profesional (Rosse \& Krebs, 1999; Susilo et al. 2014). Por lo tanto, el paciente debe estar libre de imposiciones por parte de los profesionales de la salud, por lo que un aspecto importante dentro del rol sería asegurar que no se va a proceder con la intervención si el paciente no comprende plenamente, asegurando que no se sienta intimidado o presionado por el entorno (Sood \& Gupta, 2018). Estos planteamientos toman mayor peso en el caso de adultos mayores, niños u otras comunidades, quienes podrían no entender la información brindada, pero deciden hacer parte de la intervención o tratamiento, por la confianza en el profesional.

Es necesario recordar que el no obtener un consentimiento informado antes de proceder es una violación a los derechos del paciente y esto trae repercusiones éticas y legales. El consentimiento no debe ser considerado como un proceso rígido que requiere de la divulgación estricta de una gran cantidad de información, por lo tanto, se debe considerar un proceso flexible y la cantidad requerida 
es la que facilita la toma de decisiones por parte del paciente, haciendo énfasis en que el CI requiere una información mínima, pero no siempre es la necesaria, porque cada paciente y sus necesidades son diferentes, de acuerdo con el nivel de riesgo de la investigación o intervención. En este sentido, la cantidad y la profundidad de la información contenida varía y, por lo tanto, se debe adaptar a las necesidades individuales de las personas (Aveyard, 2005).

Como lo propone Álvarez (2015), existe la necesidad de comprender el concepto de autonomía, por tradición centrado en el individuo y ampliarlo al sistema de relaciones de las personas, lo que conlleva, como anota la autora, al concepto de autonomía relacional. El concepto de autonomía, que guía la práctica clínica (relational autonomy, in-control agent), da cuenta del posicionamiento que toma el profesional en la relación. Entendido el CI, como un fenómeno relacional y, la autonomía, como una experiencia individual y colectiva, el problema para el área de la salud iría más allá de respetar o no la decisión del paciente, en un momento dado y se centra en la construcción de esa experiencia (Walter \& Ross, 2014).

En ese sentido, las decisiones en salud tienen como fin último no hacer daño y mejorar la condición de salud de las personas, por lo tanto, la autonomía del paciente tendría que dar cuenta del profesional del área de la salud y del sistema de salud en general, como elementos importantes en el posicionamiento del paciente. Desde esta lógica, la responsabilidad del profesional del área de la salud iría más allá de dar información acerca del procedimiento a realizar para obtener una firma.

Incluir al profesional de la salud como parte del fenómeno, conlleva a la necesidad de preguntarse: para el profesional ¿qué es autonomía? y para él ¿cuál es la intención de la firma del consentimiento informado? Si la intención del profesional es tener una especie de seguro ante futuras implicaciones legales, con la obtención de la firma sería suficiente; sin embargo, si la intención es comprender con el paciente las posibles implicaciones, favorables o no del procedimiento en su contexto particular para que al final tome una decisión, la firma del consentimiento, no sería suficiente.

En ese sentido, como lo proponen Rathor et al. (2016), la promoción de la autonomía no implica ignorar la pericia y la experiencia del investigador clínico, sino que implica su habilidad para facilitar la toma de decisiones autónomas, según las necesidades de los pacientes. Además, como anota Osuji (2018), el concepto de autonomía relacional en el contexto del CI está más cerca de la experiencia humana y de una verdadera ética del cuidado.

Para concluir, la obtención del CI, se debería plantear a través del diálogo entre el profesional y el paciente o participante en la investigación acerca del procedimiento que se va a realizar, sus expectativas, los riesgos y beneficios desde los saberes y en las condiciones contextuales particulares de las personas, formalizado a través de la firma de un documento, donde se consta que se comprendió la información brindada y se decide participar en el estudio o recibir la intervención o el procedimiento planteado. Para lograr este propósito, se necesita un cambio en la comprensión del CI, como limitado a los contextos jurídicos, que le dieron origen y del ser humano y, por tanto, del quehacer profesional, desde su complejidad y multidimensionalidad.

Surge el desafío para los profesionales de la salud, para mejorar la comprensión del CI, desafío que, desde la práctica, la investigación y los comités de ética, se debe abordar para aportar a la solución de esta problemática. Dentro de esta publicación y como resultado, se adjunta la proforma de consentimiento informado, diseñado por el Comité de Ética en Investigación en Humanos de la Facultad de Ciencias de la Salud (CEIHFCS), Universidad de Ciencias Aplicadas y Ambientales U.D.C.A (Cuadro 1); sin embargo, debe ser parte de un proceso dialógico entre el profesional y el participante en el estudio.

Cuadro 1. Proforma de consentimiento informado.

\section{CONSENTIMIENTO INFORMADO PARA PARTICIPAR EN EL ESTUDIO DE INVESTIGACION "Titulo del proyecto"}

Por favor lea atentamente este documento, el cual, le brindará la información necesaria para ayudarle a decidir sobre su participación en este estudio, sino no le queda claro, le surge alguna duda o pregunta o desea información adicional, por favor sírvase hacerlo saber a alguno de los investigadores, que se mencionan a continuación de este documento.

1. ¿Por qué se realiza esta investigación, cuánto tiempo durará y número de personas participantes? Su participación es de gran importancia, gracias a ella, se permitirá conocer e investigar sobre (objetivo del estudio). Se espera que alrededor de unas (\#) personas participarán y el estudio va durar (tiempo en meses).

2. ¿En qué consiste la investigación? Explicar de manera clara, corta y precisa la metodología del estudio, evitando usar lenguaje técnico, siglas o abreviaturas.

3. ¿Quién está a cargo de la investigación? ¿Quiénes son los investigadores? ¿Dónde los puedo contactar? Nombre del director de la investigación, nombres de los co-investigadores, correo electrónico, de ser posible un número de contacto y filiación de los investigadores con dirección. 
Continuación cuadro 1.

4. ¿Cuáles son los efectos colaterales, riesgos y beneficios de la investigación para mí, mi familia y la comunidad? Este estudio no presenta ningún riesgo para su salud, ni efectos secundarios que pongan en riesgos su vida a corto, mediano o largo plazo (o incluir los riesgos y los efectos secundarios). Los beneficios (incluir beneficios).

5. ¿Cuánto debo pagar por participar en la investigación y que compensación voy a recibir? No tendrá ningún valor económico participar ni los exámenes, pruebas o intervenciones que se le hagan dentro de la investigación, de igual manera, no se le dará dinero por participar en la investigación.

6. ¿Cómo me van a garantizar que no se revelará mi identidad y que no van a dar información con la que me puedan identificar? En este aparte, los investigadores deben indicar el manejo que se dará a los datos recolectados, para garantizar que no se revele la identidad de las personas que hacen parte del estudio.

7. ¿Para qué sirven los resultados de esta investigación? En este aparte, se recomienda que los investigadores den cuenta de los objetivos de la investigación y de la utilidad de los posibles resultados.

8. ¿Cuáles son mis derechos como participante, o si decido no participar o cambiar de opinión después de haber firmado que acepto? En este aparte debe quedar que el hecho de que firme el consentimiento no implica que debe continuar en la investigación hasta el final. En otras palabras, debe quedar claro que tiene la libertad de retirarse del estudio en el momento que quieran y esto no tendrá ninguna consecuencia ética ni jurídica.

9. ¿Existe alguna normatividad vigente relacionada con las investigaciones que protejan mis derechos? Se recomienda que en esta respuesta los investigadores informen la norma vigente que regula la investigación, por ejemplo, la resolución 8430 de 1993 del Ministerio de Salud. Además, deben informar el número de acta del Comité de Investigación y de Ética que aprobó el proyecto de investigación. Debe quedar claro en la respuesta que la investigación cumple con los lineamientos vigentes de ética en investigación.

10. ¿Quiénes pueden contestar mis preguntas, resolver mis dudas o si tengo algún problema relacionado con el estudio? Si usted requiere aclaración adicional sobre sus derechos como participante en esta investigación o cree que alguno de sus derechos ha sido vulnerado, puede escribir al Comité de Ética en Investigación (nombre del comité de ética, del presidente del mismo y datos de contacto).

\section{Declaración de consentimiento informado}

Se recomienda que, en este aparte, los investigadores dejen un texto donde si informa a la persona que si decide participar en la investigación lo debe firmar, en caso contrario, no debe firmarlo. Además, se debe indicar que se le entrega una copia del consentimiento informado al participante.

Firmas

Incluir:

Nombres y Apellidos completos, número y tipo de documento de identidad, firma y número telefónico del participante y dos (2) testigos. Nombre y Apellidos completos, número y tipo de documento y firma del investigador principal de investigador principal. Ciudad y fecha.

Conflictos de interés: El manuscrito fue preparado y revisado con la participación de todos los autores, quienes declaramos que no existe conflicto de intereses que ponga en riesgo la validez de los resultados presentados. Parte de la información reportada, se deriva del trabajo de grado de la segunda autora y dirigido por el primer autor "Rol de enfermería en la aplicación y entendimiento del consentimiento informado" y disponible en: https:/ / repository. udca.edu.co/handle/11158/921.

\section{REFERENCIAS}

1. AL-SAADOON, M.; AL-ADAWI, S. 2019. Informed Consent in societies with different ethos of 'selfhood'. Sultan Qaboos Univ Med J. 19(1): e1. https://doi.org/10.18295/ squmj.2019.19.01.001
2. ÁLVAREZ, S. 2015. La autonomía personal y la autonomía relacional. Anal. filos. 35(1):13-26.

3. ANNAS, G.J. 2018. Beyond Nazi war crimes experiments: the voluntary consent requirement of the Nuremberg Code at 70. Am J Public Health. 108(1):42-46. https://doi. org/10.2105/AJPH.2017.304103

4. AVEYARD, H. 2005. Informed consent prior to nursing care procedures. Nurs Ethics. 12(1):19-29. https://doi. org/10.1191/0969733005ne755oa

5. BEAUCHAMP, T.L.; CHILDRESS, J.F. 2009. Principles of biomedical ethics. Oxford University Press, USA. 417p. 
6. BESKOW, L.M.; FRIEDMAN, J.Y.; HARDY, N.C.; LIN, L.; WEINFURT, K.P. 2010. Developing a simplified consent form for biobanking. PLoS One. 5(10):e13302. https:// doi.org/10.1371/journal.pone.0013302

7. BOGOD, D. 2004. The Nazi hypothermia experiments: forbidden data? Anaesthesia. 59(12):1155-1156. https:// doi.org/10.1111/j.1365-2044.2004.04034.x

8. BREESE, P.E.; BURMAN, W.J.; GOLDBERG, S.; WEIS, S.E. 2007. Education level, primary language, and comprehension of the informed consent process. J Empir Res Hum Res Ethics. 2(4):69-79. https://doi. org/10.1177/1556264619831888

9. CAÑETE, R.; GUILÑHEM, D.; BRITO, K. 2012. Consentimiento informado: algunas consideraciones actuales. Acta Bioethica. 18(1):121-127. https://doi. org/10.4067/S1726-569X2012000100011

10. CARREÑO-DUEÑAS, J.A. 2016. Consentimiento informado en investigación clínica: un proceso dinámico. Pers.bioét. 20(2):232-243. https://doi.org/10.5294/pebi.2016.20.2.8

11. CHUAIRE, L.; SÁNCHEZ, M.C. 2007. Platón y el consentimiento informado contemporáneo. Colomb. Med. 38(3):297-300.

12. CLIFFORD, T. 2013. Informed consent-role of the perianesthesia nurse. J. Perianesth. Nurs. 28(6):413-414. https://doi.org/10.1016/j.jopan.2013.09.004

13. DE LOS DERECHOS HUMANOS, Declaración Universal. Organización de las Naciones Unidas. Asamblea General del. 1948. 10.

14. DHAI, A. 2014. The research ethics evolution: From Nuremberg to Helsinki. S Afr Med J. 104(3):178-180. http://dx.doi. org/10.7196/SAMJ.7864

15. ELLETT, L.; VILLEGAS, R.; BEISCHER, A.; ONG, N.; MAHER, P. 2014. Use of a multimedia module to aid the informed consent process in patients undergoing gynecologic laparoscopy for pelvic pain: randomized controlled trial. J. Minim. Invasive Gynecol. 21(4):602611. http://dx.doi.org/10.1016/j.jmig.2014.01.002

16. ESCOBAR LÓPEZ, M.T.; CARRERA CELIS, C.A. 2015. Percepción sobre consentimiento informado en pacientes de cuatro diferentes áreas de atención en salud en Bogotá, Colombia. Rev. Latinoam. Bioet. 15(1):14-25.

17. ESCUDERO CARRETERO, M.J.; GARCÍA TOYOS, N.; MARTÍN BARATO, A.I.; RUIZ AZAROLA, A.; PÉREZ CORRAL, O.; PRIETO RODRÍGUEZ, M.A.; SUESS
SCHWEND, A. 2017. Valoración de aspectos éticos y metodológicos en un estudio cualitativo con personas usuarias de Oncología Pediátrica. EMPIRIA. 36:149-176. https://doi.org/10.5944/empiria.36.2017.17863

18. FAIRCHILD, A.L.; BAYER, R. 1999. Uses and abuses of Tuskegee. Science. 284(5416):919-921. https://doi. org/10.1126/science.284.5416.919

19. FERNANDES, N.C. 2015. The writing of informed consent in accessible language: Difficulties. Rev. Colégio Bras. Cirurgiões. 42(3):197-199. http://dx.doi. org/10.1590/0100-69912015003013

20. FLORY, J.; EMANUEL, E. 2004. Interventions to improve research participants' understanding in informed consent for research: a systematic review. JAMA. 292(13):15931601. https://doi.org/10.1001/jama.292.13.1593

21. GONZÁLEZ-LÓPEZ, E.; RÍOS-CORTÉS, R. 2016. Visiting holocaust-related sites with medical students as an aid in teaching medical ethics. IMAJ. 18(5):257-260.

22. GROOTENS-WIEGERS，P.; DE VRIES，M.C.; VAN BEUSEKOM, M.M.; VAN DIJCK, L.; VAN DEN BROEK, J.M. 2015. Comic strips help children understand medical research: targeting the informed consent procedure to children's needs. Patient Educ. Couns. 98(4):518-524. https://doi.org/10.1016/j.pec.2014.12.005

23. GUIX OLIVER, J.; BALAÑ̀̀ FORT, L.; CARBONELL RIERA, J.M.; SIMÓN PÉREZ, R.; SURROCA MACIÀ, R.M.; NUALART BERBEL, L. 1999. Cumplimiento y percepción del consentimiento informado en un sector sanitario de Cataluña. Rev. Esp. Salud Pública. 73:669-675.

24. HADZIABDIC, E.; HEIKKILÄ, K.; ALBIN, B.; HJELM, K. 2011. Problems and consequences in the use of professional interpreters: qualitative analysis of incidents from primary healthcare. Nurs Inq. 18(3):253-261. https:/ / doi.org/10.1111/j.1440-1800.2011.00542.x

25. HALKOAHO, A.; PIETILÄ, A.M.; EBBESEN, M.; KARKI, S.; KANGASNIEMI, M. 2016. Cultural aspects related to informed consent in health research: A systematic review. Nurs. Ethics. 23(6):698-712.

26. HOOVER-REGAN, M.; BECKER, T.; WILLIAMS, M.J.; SHENKER, Y. 2013. Informed consent and research subject understanding of clinical trials. WMJ. 112(1):18-23.

27. HUNT, L.M.; DE VOOGD, K.B. 2007. Are good intentions good enough? Informed consent without trained interpreters. J. Gen. Intern. Med. 22(5):598-605. https:// doi.org/10.1007/s11606-007-0136-1 
28. KARAN, A.; SOMASUNDARAM, P.; MICHAEL, H.; SHAYEGANI, A.; MAYER, H. 2014. The effect of multimedia interventions on the informed consent process for cataract surgery in rural South India. Indian J. Ophthalmol. 62(2):171-175. https://doi. org/10.4103/0301-4738.116488

29. KARLINER, L.S.; JACOBS, E.A.; CHEN, A.H.; MUTHA, S. 2007. Do professional interpreters improve clinical care for patients with limited English proficiency? A systematic review of the literature. Health Serv Res. 42(2):727-754. https://doi.org/10.1111/j.1475-6773.2006.00629.x

30. KATZ, J. 1996. The Nuremberg code and the Nuremberg trial: A reappraisal. JAMA. 276(20):1662-1666. https:// doi.org/10.1001/jama.1996.03540200048030

31. KHABOUR, O.F.; ALOMARI, M.A.; AL-SHEYAB, N.A. 2017. Parental perceptions about informed consent/ assent in pediatric research in Jordan. J. Empir. Res. Hum. Res. Ethics. 12(4):261-268. https://doi. org $/ 10.1177 / 1556264617718937$

32. KOT'TOW, M. 2014. De Helsinki a Fortaleza: una Declaración desangrada. Rev. Bioét. 22(1):28-33.

33. KOT'TOW, M. 2016. El consentimiento informado en clínica: inquietudes persistentes. Rev. Med. Chile. 144(11):14591463. https://doi.org/10.4067/S0034-98872016001100012

34. LAD, P.M.; DAHL, R. 2014. Overcoming language barriers in the informed consent process: Regulatory and compliance issues with the use of the "Short Form". Accountability in Research Policies and Quality Assurance. 21(5):315-320. https://doi.org/10.1080/08989621.2013.848801

35. LIN, Y.K.; LIU, K.T.; CHEN, C.W.; LEE, W.C.; LIN, C.J.; SHI, L.; TIEN, Y.C. 2019. How to effectively obtain informed consent in trauma patients: a systematic review. BMC medical ethics. 20(1):8. https://doi.org/10.1186/s12910019-0347-0

36. LÓPEZ-MUÑOZ, F; ALAMO, C.; GARCÍA-GARCÍA, P.; MOLINA, J.D.; RUBIO, G. 2008. The role of psychopharmacology in the medical abuses of the Third Reich: From euthanasia programmes to human experimentation. Brain Res. Bull. 77(6):388-403. https:// doi.org/10.1016/j.brainresbull.2008.09.002

37. MALIK, L.; MEJIA, A. 2014. Informed consent for phase I oncology trials: form, substance and signature. J. Clin Med Res. 6(3):205-208. https://doi.org/10.14740/jocmr1803w

38. MANZINI, J.L. 2000. Declaración de Helsinki: principios éticos para la investigación médica sobre sujetos humanos. Acta Bioethica. 6(2):321-334.
39. MENENDEZ, J.B. 2013. Informed consent: essential legal and ethical principles for nurses. JONA'S healthcare law, ethics regulation. 15(4):140-144. https://doi.org/10.1097/ NHL.0000000000000015

40. NELSON-MARTEN, P.; RICH, B.A. 1999. A historical perspective of informed consent in clinical practice and research. Semin. Oncol. Nurs. 15(2):81-88. https://doi. org/10.1016/S0749-2081(99)80065-5

41. OSUJI, P.I. 2018. Relational autonomy in informed consent (RAIC) as an ethics of care approach to the concept of informed consent. Med Health Care and Philos. 21(1):101111. https://doi.org/10.1007/s11019-017-9789-7

42. PORRINO, P.; FALCONE, Y.; AGOSTA, L.; ISAIA, G.; ZANOCCHI, M.; MASTRAPASQUA, A.; ISAIA, G.; BO, M. 2015. Informed Consent in Older Medical Inpatients: Assessment of Decision-Making Capacity. J. Am. Geriatr. Soc. 63(11):2423-2424. https://doi.org/10.1111/jgs.13810

43. POTTER, V.R. 1970. Bioethics, the science of survival. Perspect. Biol. Med. 14(1):127-153. https://doi.org/10.1353/ pbm.1970.0015

44. PRUSACZYK, B.; CHERNEY, S.M.; CARPENTER, C.R.; DUBOIS, J.M. 2017. Informed consent to research with cognitively impaired adults: transdisciplinary challenges and opportunities. Clinical Gerontologist. 40(1):63-73. https:// doi.org/10.1080/07317115.2016.1201714

45. RATHOR, M.Y.; SHAH, A.; HASMONI, M.H. 2016. Is Autonomy a Universal Value of Human Existence? Scope of Autonomy in Medical Practice: A Comparative Study between Western Medical Ethics and Islamic Medical Ethics. Int. Med. J. 15(1):81-88.

46. REVERBY, S.M. 2016. Restorative justice and restorative history for the sexually transmitted disease inoculation experiments in Guatemala. Am. J. Public Health. 106(7):1163-1164. https://doi.org/10.2105/AJPH.2016.303205

47. RICE, T.W. 2008. The historical, ethical, and legal background of human-subjects research. Respir Care. 53(10):1325-1329.

48. RODRÍGUEZ, M.A.; GARCÍA, R. 2013. First, do no harm: the US sexually transmitted disease experiments in Guatemala. Am J Public Health, 103(12):2122-2126. https://doi. org/10.2105/AJPH.2013.301520

49. ROGERS, C.G.; TYSON, J.E.; KENNEDY, K.A.; BROYLES, R.S.; HICKMAN, J.F. 1998. Conventional consent with opting in versus simplified consent with opting out: an exploratory trial for studies that do not increase patient risk. J. Pediatr. 132(4):606-611. https://doi.org/10.1016/ S0022-3476(98)70347-6 
50. ROSSE, P.A.; KREBS, L.U. 1999. The nurse's role in the informed consent process. Semin. Oncol. Nurs. 15(2):116123. https://doi.org/10.1016/S0749-2081(99)80069-2

51. SABBATANI, S. 2013. The infectious diseases experiments conducted on human guinea pigs by Nazis in concentration camps. Infez. Med. 21(2):151-166.

52. SANDVIK, A.; LIE, A.K. 2016. Untreated syphilis-from Oslo to Tuskegee. Tidsskr Nor Laegeforen. 136(23-24):20102016. https://doi.org/10.4045/tidsskr.16.0543

53. SASS, H.M. 2007. Fritz Jahr's 1927 concept of bioethics. Kennedy Inst Ethics J. 17(4):279-295. https://doi. org/10.1353/ken.2008.0006

54. SILVER, J.R. 2011. Karl Gebhardt (1897-1948): A lost man. J. R. Coll. Physicians Edinb. 41(4):366-371. https://doi. org/10.4997/JRCPE.2011.417

55. SIMS, J.M. 2010. A brief review of the Belmont report. Dimens. Crit, Care Nurs. 29(4):173-174.

56. SIMKULET, W. 2017. Informed consent and nudging. Bioethics. 333:169-184. https://doi.org/10.1111/bioe.12449

57. SOOD, A.; GUPTA, J. 2018. Patient counselling and consent. Best Pract. Res. Clin. Obstet. Gynaecol. 46:43-47. https:/ / doi.org/10.1016/j.bpobgyn.2017.10.002

58. SPENCER, S.P.; STONER, M.J.; KELLEHER, K.; COHEN, D.M. 2015. Using a multimedia presentation to enhance informed consent in a pediatric emergency department. Pediatr Emerg Care. 31(8): 572-576. https://doi. org/10.1097/PEC.0000000000000513

59. SSALI, A.; POLAND, F.; SEELEY, J. 2015. Volunteer experiences and perceptions of the informed consent process: Lessons from two HIV clinical trials in Uganda.
BMC Med Ethics. 16(1):86. https://doi.org/10.1186/ s12910-015-0073-1

60. SUÁREZ-OBANDO, F. 2016. Consentimiento informado como criterio de inclusión ¿confusión conceptual, manipulación, discriminación o coerción? Pers. Bioét. 20(2):244-256. http://dx.doi.org/10.5294/PEBI.2016.20.2.9

61. SUSILO, A.P.; DALEN, J.V.; CHENAULT,M.N.; SCHERPBIER, A. 2014. Informed consent and nurses' roles: a survey of Indonesian practitioners. Nurs. Ethics. 21(6):684-694. https://doi.org/10.1177/0969733014531524

62. SYNNOT, A.; RYAN, R.; PRICTOR, M.; FETHERSTONHAUGH, D.; PARKER, B. 2014. Audiovisual presentation of information for informed consent for participation in clinical trials. Cochrane Database Syst Rev. 9(5):CD003717. https://doi.org/10.1002/14651858. CD003717.pub3

63. VERA CARRASCO, O. 2016. El consentimiento informado del paciente en la actividad asistencial médica. Rev. Méd. La Paz. 22(1):59-68.

64. VOLLMANN, J.; WINAU, R. 1996. Informed consent in human experimentation before the Nuremberg code. BMJ. 313(7070):1445-1449. https://doi.org/10.1136/ bmj.313.7070.1445

65. WALTER, J.K.; ROSS, L.F. 2014. Relational autonomy: moving beyond the limits of isolated individualism. Pediatrics. 133(Supplement 1):S16-S23. https://doi.org/10.1542/ peds.2013-3608D

66. WEST, E.; STUCKELBERGER, A.; PAUTEX, S.; STAAKS, J.; GYSELS, M. 2017. Operationalising ethical challenges in dementia research - a systematic review of current evidence. Age Ageing. 46(4):678-687. https://doi. org/10.1093/ageing/afw250 